\title{
Pulmonary infection with Nocardia species: a report of 10 cases and review
}

\author{
R. Menéndez*, P.J. Cordero*, M. Santos**, M. Gobernado**, V. Marco*
}

Pulmonary infection with Nocardia species: a report of 10 cases and review. R. Menéndez, P.J. Cordero, M. Santos, M. Gobernado, V. Marco. CERS Journals Ltd 1997.

ABSTRACT: Pulmonary nocardiosis $(P N)$ is an infrequent and severe infection due to Nocardia spp., microorganisms that may behave both as opportunists and as primary pathogens. The aim of this study and review was to evaluate the clinical features, evolution and prognostic factors of PN.

The study group comprised 10 consecutive patients with pulmonary nocardiosis acquired in a community setting, diagnosed and followed in a tertiary teaching hospital.

Chronic obstructive pulmonary disease (COPD), neoplastic disease and human immunodeficiency virus (HIV) infection were the most frequent predisposing factors. Four patients were receiving corticosteroid treatment. Clinical course was chronic and diagnosis was delayed 3 weeks or more in seven of the patients. Lobar or multilobar condensation was the most frequent radiographic pattern. Antimicrobial susceptibility testing showed: $100 \%$ sensitivity for amikacin; $83 \%$ for imipenem; $71 \%$ for cefotaxime; and $71 \%$ for trimethoprim-sulphamethoxazole. The disease remained localized in the lung in five cases, with a trend toward chronicity in one with bronchiectasis. In the other five, the disease disseminated, affecting subcutaneous tissue, the central nervous system and the kidney. Three patients died, one with disseminated disease and two who were receiving corticosteroid therapy.

The following conclusions were reached: 1) pulmonary nocardiosis is difficult to diagnose, diagnosis is frequently delayed and a high level of suspicion is, thus, required in patients with underlying diseases or chronic corticosteroid therapy; 2) there is frequent dissemination and high mortality; and 3) antimicrobial combinations with proven synergy, such as imipenem and amikacin, are recommended for initial therapy.

Eur Respir J 1997; 10: 1542-1546
*Pneumology Service and **Microbiology Service, University Hospital La Fe, Valencia, Spain.

Correspondence: R. Menéndez

Servicio de Neumología

Hospital La Fe

Avda. de Campanar 21

46009 Valencia

Spain

Keywords: Bacterial pneumonia nocardiosis

pulmonary nocardiosis

Received: October 211996

Accepted after revision April 141997
Pulmonary nocardiosis (PN) is an infrequent but severe infection that commonly presents as a subacute or chronic suppurative disease, mimicking a lung carcinoma or abscess. Nocardia spp. are aerobic Gram-positive bacteria of the order Actinomycetales. In humans, $N$. asteroides complex is the predominant pathogen, but there are several other species, including: $N$. brasiliensis and $N$. otitidiscaviarum [1]. Pulmonary infection is usually produced by $N$. asteroides (85\%), whereas $N$. brasiliensis causes cutaneous and subcutaneous abscesses [2].

Nocardia most often enters through the respiratory tract, and is able to produce infection in a host compromised by systemic disease or anti-inflammatory therapy $(50 \%)$; however, it can also produce infection in patients with no concurrent abnormality [2-41.

In Spain, no information about annual incidence is available, but in the USA there are an estimated 500-1,000 new cases each year. It is considered that the incidence of this infection has increased since 1960 [2]. The reason for this increasing incidence is unknown, but may be due to improved and more aggressive diagnostic tests, a raised suspicion, and the growing use of immunosuppressive therapy [5].
Experience of pulmonary nocardiosis in the medical literature is limited to case reports, with few series, the latter also including extrapulmonary nocardiosis. The aim of the present review was to evaluate the clinical features, evolution and prognostic factors in a series of 10 cases of nocardiosis with pulmonary involvement.

\section{Patients and methods}

The reports on 10 patients diagnosed with pulmonary nocardiosis in our hospital (a tertiary-care teaching hospital) from January 1989 to May 1994 were reviewed. The accepted inclusion criteria were the presence of Nocardia spp. in samples obtained by fibreoptic bronchoscopy from the respiratory tract, from the pleural cavity, or from at least two different sputum cultures. Patients with extrapulmonary nocardial infection but with no evidence of pulmonary disease were excluded.

The following data were collected: predisposing factors; clinical manifestations; radiographic findings; bacteriological reports; treatment; outcome of illness; duration of infection from the onset of symptoms to diagnosis; 
and, eventually, death and cause of death. The extent of disease was divided into two categories: localized or disseminated. Localized infection was confined to one organ. Disseminated nocardiosis was defined as infection in two noncontiguous organs or in the central nervous system [5].

\section{Results}

\section{Characteristics and clinical features of the patients}

From January 1989 to May 1994, 18 patients were diagnosed with nocardial infection. Eight patients were excluded because of nonpulmonary disease (five in subcutaneous tissue, two with brain abscess associated with subcutaneous tissue infection, and one with renal involvement). Finally, 10 patients were included (seven males and three females), with a mean age of 53 yrs (range: 18-73 yrs). Characteristics of the patients, with demographic data, concurrent illnesses, prior treatment and analytical results, are detailed in table 1 . The following diseases were associated in 8 of the 10 patients: chronic obstructive pulmonary disease (3); human immunodeficiency virus (HIV) infection (2); neoplastic disease (2); alcoholism (1); and bronchiectasis (1). Three of the patients were receiving corticosteroid therapy, and one patient was receiving corticosteroid and immunosuppressive therapy (cisplatin +5 -fluorouracil). HIV-positive patients were staged according to the Centers for
Disease Control and Prevention (CDC) 1986 classification (patient No. 4: group IV with CD4+ T-lymphocyte count of 12 cells $\cdot \mathrm{mm}^{-3}$; patient No. 8: group II with $\mathrm{CD} 4+$ of 756 cells $\cdot \mathrm{mm}^{-3}$ ).

The clinical manifestations in the 10 patients were: cough (10); purulent expectoration (7); and fever (7). In general, the clinical course was chronic, with a duration of symptoms before diagnosis of 3 weeks or more in 7 of the 10 (range 1 week to 3 months). Absolute and differential white blood cell (WBC) counts and erythrocyte sedimentation rate (ESR) at diagnosis are presented in table 1 .

\section{Radiographic patterns}

The radiographic picture of the 10 patients included several manifestations: lobar or multilobar consolidation (7); solitary masses (2); and reticulonodular infiltrate (1). Pleural effusion occurred in three of the 10 patients, and cavitary lesion in three. The upper lobes were involved in $70 \%$. Unilateral disease was noted in six patients and bilateral in four. The progression of radiographic abnormalities was slow.

\section{Microbiological identification}

Microbiological diagnosis was made in all patients by isolation of Nocardia spp. from respiratory samples (table 2): sputum cultures in 9 out of 10; bronchoalveolar lavage

Table 1. - Clinical characteristics and analytical results

\begin{tabular}{|c|c|c|c|c|c|c|c|c|}
\hline $\begin{array}{l}\text { Case } \\
\text { No. }\end{array}$ & $\begin{array}{l}\text { Age } \\
\text { yrs }\end{array}$ & Sex & Concurrent illness & Steroids & $\begin{array}{c}\text { WBC } \\
\times 10^{9} \cdot \mathrm{L}^{-1}\end{array}$ & $\begin{array}{l}\text { Differential } \\
\text { PMNs }\end{array}$ & $\begin{array}{l}\text { count \% } \\
\text { Lym }\end{array}$ & $\begin{array}{c}\text { ESR } \\
\mathrm{mm} \cdot \mathrm{h}^{-1}\end{array}$ \\
\hline 1 & 71 & M & Vesicular carcinoma & Prednisone $10 \mathrm{mg} \cdot \mathrm{day}^{-1}$ for 9 months & 17.0 & 75 & 18 & 94 \\
\hline 2 & 65 & M & Bronchogenic carcinoma & Dexamethasone $8 \mathrm{mg} \cdot \mathrm{day}^{-1}$ for 60 days & 7.8 & 81 & 12 & 33 \\
\hline 3 & 18 & $\mathrm{~F}$ & - & - & 9.9 & 73 & 17 & 124 \\
\hline 4 & 20 & M & HIV infection & - & 4.4 & 77 & 10 & 70 \\
\hline 5 & 73 & M & COPD & - & 29.0 & 94 & 1 & 116 \\
\hline 6 & 56 & M & Alcoholism & - & 10.7 & 88 & 5 & 23 \\
\hline 7 & 64 & M & COPD, bronchiectasis & - & 8.6 & 58 & 26 & 45 \\
\hline 8 & 33 & M & HIV infection & Prednisone $40 \mathrm{mg} \cdot \mathrm{day}^{-1}$ for 15 days & 7.7 & 78 & 16 & 27 \\
\hline 9 & 50 & $\mathrm{~F}$ & - & - & 3.9 & 53 & 36 & 20 \\
\hline 10 & 70 & $\mathrm{~F}$ & COPD & Prednisone $30 \mathrm{mg} \cdot \mathrm{day}^{-1}$ for 21 days & 34.4 & 63 & 24 & 60 \\
\hline
\end{tabular}

M: male; F: female; WBC: white blood cell count; PMNs: polymorphonuclear neutrophils; Lym: lymphocytes; ESR: erythrocyte sedimentation rate; HIV: human immunodeficiency virus; COPD: chronic obstructive pulmonary disease.

Table 2. - Sources of positive cultures, treatment and outcome

\begin{tabular}{|c|c|c|c|c|}
\hline $\begin{array}{l}\text { Case } \\
\text { No. }\end{array}$ & Site of cultures & Treatment & $\begin{array}{c}\text { Duration } \\
\text { weeks }\end{array}$ & Outcome \\
\hline 1 & BAL, sputum & $\begin{array}{l}\text { Imipenem } \\
\text { TMP-SMX }\end{array}$ & $\begin{array}{r}3 \\
26\end{array}$ & Localized (resolution) \\
\hline 2 & Sputum, abscess puncture & TMP-SMX & 2 & Dissemination to ST (death) \\
\hline 3 & Pleural fluids, sputum & Ciprofloxacin & 17 & Localized (resolution) \\
\hline 4 & Sputum, abscess puncture & TMP-SMX & 26 & Dissemination to ST (resolution) \\
\hline 5 & Sputum & Amoxicillin-clavulanic & 26 & Localized (resolution) \\
\hline 6 & Sputum, abscess puncture & TMP-SMX & 2 & Dissemination to CNS (death) \\
\hline 7 & Sputum, BW & $\begin{array}{l}\text { Netilmicin } \\
\text { ciprofloxacin }\end{array}$ & $\begin{array}{r}3 \\
22\end{array}$ & Localized (chronic infection) \\
\hline 8 & Pleural fluid, pleural biopsy & $\begin{array}{l}\text { Imipenem, } \\
\text { TMP-SMX }\end{array}$ & $\begin{array}{r}3 \\
26\end{array}$ & Dissemination to ST (resolution) \\
\hline 9 & Sputum, BAL, BW, urine & Ciprofloxacin & 13 & Dissemination to kidney (resolution) \\
\hline 10 & Sputum, BAL, BW, BC & $\begin{array}{l}\text { Imipenem } \\
\text { TMP-SMX }\end{array}$ & $\begin{array}{l}3 \\
3\end{array}$ & Localized (death) \\
\hline
\end{tabular}

TMP-SMX: trimethoprim-sulphamethoxazole; BAL: bronchoalveolar lavage; BW: bronchial washings; BC: brush catheter; CNS: central nervous system; ST: subcutaneous tissue. 
in 3 out of 3; brush catheter in 2 out of 3; pleural fluid in 2 out of 2; and pleural biopsy in 1 out of 2 . In four patients, Nocardia spp. were also isolated in other samples (abscess puncture in three and urine in one). In all patients, except case No. 5, Nocardia spp. were isolated in sputum and other samples. In patient No. 5, Nocardia spp. were repeatedly isolated from sputum (three stains and three cultures), no other microorganisms were found and, after treatment, eradication of bacteria was achieved. No other co-pathogens were found, except for case No. 8 in which S. epidermidis was also cultured in pleural fluid. Microbiological identification was attained using standard methods [6]. Samples were examined by direct microscopic observation of preparations with Gram, acid-fast and weakly acid-fast stains. All specimens were cultured on blood agar plates, brain-heart infusion agar and blood-chocolate plates. Nocardia spp. was identified using manual and automated (Vitek System; bio-Mérieux, SA, Marcy-l'Etoile, France) standard methods. Nocardia asteroides complex was identified in eight patients. Susceptibilities were performed on several antimicrobial agents by disk diffusion in blood agar plates and Mueller-Hinton agar, as recommended by the National Committee for Clinical Laboratory Standards (NCCLS) [6, 7]. The results were read after 24, 48 and $72 \mathrm{~h}$. The antibiotic susceptibility pattern is presented in table 3 .

\section{Clinical outcome}

Resolution of the disease was defined as eradication of Nocardia spp., together with clinical and radiological improvement. Pulmonary nocardiosis resolved in six patients (case Nos. 1, 3, 4, 5, 8 and 9), became chronic in one patient (case No. 7), and three patients died. The clinical end-points were freedom from fever, which was achieved during the first days (range 2-5 days) after treatment, with reduction in purulence of sputum and WBC counts. Radiological improvement lagged behind clinical parameters, with complete resolution in 1-3 months (patient Nos. 1, 3, 4, 8 and 9), and partial resolution in patient No. 5 .

The disease was localized in five (case Nos. 1, 3, 5, 7 and 10) of the 10 patients (table 2). One of these

Table 3. - In vitro test of antibiotic sensitivity

\begin{tabular}{|c|c|c|c|c|c|c|c|c|c|c|}
\hline \multirow[t]{2}{*}{ Drug } & \multicolumn{10}{|c|}{ Case No. } \\
\hline & 1 & 2 & 3 & 4 & 5 & 6 & 7 & 8 & 9 & 10 \\
\hline Netilmicin & $\mathrm{S}$ & $\mathrm{S}$ & $\mathrm{S}$ & $\mathrm{S}$ & $\mathrm{S}$ & - & $\mathrm{S}$ & - & S & - \\
\hline Amikacin & $\mathrm{S}$ & - & $\mathrm{S}$ & $\mathrm{S}$ & $\mathrm{S}$ & - & $\mathrm{S}$ & - & S & $\mathrm{S}$ \\
\hline Imipenem & $\mathrm{S}$ & - & $\mathrm{S}$ & - & $\mathrm{S}$ & - & $\mathrm{R}$ & - & S & $\mathrm{S}$ \\
\hline Cefotaxime & $\mathrm{S}$ & - & $\mathrm{S}$ & $\mathrm{S}$ & $\mathrm{S}$ & - & $\mathrm{R}$ & - & S & $\mathrm{R}$ \\
\hline Ceftriaxone & $\mathrm{S}$ & $\mathrm{S}$ & $\mathrm{S}$ & $\mathrm{S}$ & $\mathrm{S}$ & - & $\mathrm{R}$ & - & - & $\mathrm{R}$ \\
\hline $\begin{array}{l}\text { Trimethoprim- } \\
\text { sulphamethoxazole }\end{array}$ & $\mathrm{S}$ & $\mathrm{S}$ & - & $\mathrm{S}$ & $\mathrm{R}$ & S & $\mathrm{R}$ & - & - & $\mathrm{S}$ \\
\hline Ciprofloxacin & $\mathrm{R}$ & - & $\mathrm{S}$ & $\mathrm{S}$ & $\mathrm{R}$ & - & - & - & S & $\mathrm{S}$ \\
\hline $\begin{array}{l}\text { Amoxycillin/ } \\
\text { clavulanic acid }\end{array}$ & $\mathrm{R}$ & $\mathrm{S}$ & $\mathrm{S}$ & - & $\mathrm{S}$ & - & $\mathrm{R}$ & - & $\mathrm{S}$ & $\mathrm{R}$ \\
\hline Tetracycline & $\mathrm{R}$ & $\mathrm{S}$ & $\mathrm{R}$ & $\mathrm{S}$ & $\mathrm{R}$ & - & - & - & - & $\mathrm{S}$ \\
\hline Ceftazidime & $\mathrm{R}$ & - & $\mathrm{S}$ & $\mathrm{S}$ & $\mathrm{R}$ & - & $\mathrm{R}$ & - & - & $\mathrm{R}$ \\
\hline Erythromycin & $\mathrm{R}$ & $\mathrm{S}$ & - & $\mathrm{R}$ & $\mathrm{R}$ & - & $\mathrm{R}$ & - & $\mathrm{S}$ & $\mathrm{R}$ \\
\hline Penicillin & $\mathrm{R}$ & $\mathrm{R}$ & - & $\mathrm{S}$ & $\mathrm{R}$ & - & $\mathrm{R}$ & - & - & $\mathrm{R}$ \\
\hline Aztreonam & $\mathrm{R}$ & - & $\mathrm{R}$ & $\mathrm{R}$ & $\mathrm{R}$ & - & $\mathrm{R}$ & - & - & $\mathrm{R}$ \\
\hline
\end{tabular}

S: sensitive; R: resistant. patients (patient No. 7) showed previous pulmonary disease, with a trend towards chronicity. In this case, after initial improvement with appropriate antibiotic therapy and negative findings of Nocardia spp. in sputum cultures, recurrent infection appeared, as shown by identical antibiotic susceptibility studies for almost 2 yrs and, finally, after treatment with imipenem+amikacin, eradication was achieved. One patient with localized pulmonary nocardiosis (case No. 10) died because of a respiratory distress syndrome, in spite of proven in vitro sensitivity to antimicrobial therapy; this patient was receiving corticosteroid treatment. However, the other three (case Nos. 1, 3 and 5) showed radiographic resolution and microbiological eradication of Nocardia spp. at 3 months.

In five patients, pulmonary nocardiosis disseminated to subcutaneous tissue (patient Nos. 2, 4 and 8), to the central nervous system (patient No. 6) and to the kidney (patient No. 9). Two of these patients died at 1 and 2 months of evolution (patient Nos. 2 and 6); the former was receiving corticosteroid treatment. Patients with HIV infection evolved to resolution even though the disease had disseminated to subcutaneous tissue.

Two of the four patients receiving corticosteroid treatment died, whereas only one of the six not receiving corticosteroids died.

Antibiotic therapy was started empirically, with subsequent modification according to antibiotic sensitivity tests and clinical evolution. Patient Nos. 2, 4 and 6 were treated with trimethoprim-sulphamethoxazole (TMP/ SMX), and, in spite of in vitro susceptibility, they developed a disseminated infection and two of them died. Patient Nos. 1, 8 and 10 received intravenous imipenem followed by oral TMP/SMX, with resolution at 2 months, and patient No. 10 died of respiratory distress syndrome.

\section{Discussion}

Nocardia species are common natural inhabitants of the soil throughout the world. Pulmonary nocardiosis is usually acquired by direct inhalation of Nocardia spp. from contaminated soil, and person-to-person transmission is rare. $N$. asteroides may be a saprophyte in the skin and in the upper respiratory tract. Respiratory colonization can occur, and in a compromised host it can progress to tissue invasion and dissemination [8,9]. RosetT and Hodges [10] isolated Nocardia spp. from respiratory secretions in 36 patients, 19 of whom were free of disease. Most of those colonized had obstructive pulmonary disease. Host resistance to infection with Nocardia spp. is thought to depend on functioning phagocytic cells. Neutrophils limit spread of infection in the early stage of tissue invasion [11]. Activated macrophages and T-lymphocytes prevent dissemination and kill the bacteria [12]. The crucial role of cell-mediated immunity has been proved in experimental in vitro studies; thus, it is not surprising that Nocardia spp. behaves as an opportunist microorganism in an immunocompromised host $[12,13]$. In the present study, we found underlying disease in 8 of the 10 cases of pulmonary nocardiosis: chronic obstructive pulmonary disease (COPD) (3), HIV infection (2), neoplastic disease (2) and alcoholism (1). Corticosteroid therapy was 
associated in $40 \%$ of the cases. Several studies have shown that COPD is the disease most often treated with corticosteroid therapy $[10,13,14]$. Clinical features were similar to those in previous studies. Clinical findings were nonspecific, with a chronic course in $70 \%$ before diagnosis. Leucocytes were moderately raised, with a predominance of neutrophils.

Chest radiographic manifestations were pleomorphic, as described above. In the present study, consolidation was the most frequent finding (70\%); masses appeared in $20 \%$, with a predilection for upper lobes $(70 \%)$. FEIGIN [15], in a review of 21 cases, described similar patterns and emphasized the presence of cavitation due to the necrotizing tendency of the abscesses and the association with obstructive pulmonary disease. In 21 patients with HIV infection, KRAMER and UTTAMCHANDANI [16] observed the following radiographic findings: consolidation (52\%), bilateral interstitial pattern $(33 \%)$ and solitary mass $(24 \%)$. Cavitation was associated in $62 \%$ of patients, and pleural effusion in $33 \%$. The differential diagnosis of a solitary mass in an immunocompromised host must include pulmonary nocardiosis. In patients with acquired immune deficiency syndrome (AIDS) with superior bilateral infiltrates, pulmonary nocardiosis should be taken into account because, in AIDS patients, pulmonary tuberculosis does not normally show cavitation or upper lobe lesions.

The diagnosis should always be based on isolation of Nocardia spp. in respiratory secretions. Sputum cultures were positive in $90 \%$ of the present patients, and in $100 \%$ when bronchoalveolar lavage was performed. The use of invasive diagnostic techniques improves the diagnostic yield; thus, these methods are justified when sputum fails to provide conclusive results. Isolation and identification of Nocardia spp. is troublesome. Nocardia spp. is a slowly growing microorganism that requires a prolonged period of incubation. Cultures should be maintained for at least 3 weeks before being discarded as negative. Nocardia asteroides was identified in $80 \%$ of cases. Recently, in a taxonomic revision, two new species were separated from Nocardia asteroides complex: Nocardia farcinica and Nocardia nova [1, 17]. Identification of these two new species can be achieved by gas chromatography, deoxyribonucleic acid (DNA) analysis and antimicrobial resistance patterns. Patient No. 7 showed the specific antimicrobial resistance pattern of $N$. farcini$c a$. In 347 cases reported in the USA, $N$. asteroides was responsible for $85 \%$ of cases with pulmonary involvement [2]. Although N. brasiliensis usually causes cutaneous abscesses, it was shown that it can be implicated in pulmonary nocardiosis with identical clinical features [18].

The treatment of choice for this infection includes sulphonamides and, more recently, TMP-SMX, associated with surgical drainage when required [1]. However, failure with TMP-SMX has been reported, and we observed resistance in 2 out of 7 cases (29\%). Most importantly, three patients treated with TMP-SMX that showed in vitro susceptibility developed a local spreading or disseminated infection, with a fatal outcome in two cases. Interpretation of antimicrobial testing of Nocardia spp. remains problematical [19]. Inoculum preparation, incubation of isolates and reading of inhibitory end-points may be difficult because of the characteristics of slow- er growth and tendency for elongation and branching. The NCCLS has recently organized a working group to standardize methods for susceptibility testing in the aerobic actinomycetes, including Nocardia spp.. The results of sensitivity testing do not always correlate with clinical outcome [20], so that this fatal evolution, together with the appearance of TMP-SMX-resistant Nocardia asteroides, provide a rational basis for considering combined therapy and alternative agents with a proven clinical response. It was observed that among these agents, netilmicin, amikacin and imipenem showed both in vitro susceptibility and clinical resolution [21]. Among the new $\beta$-lactams, cefmetazole has shown better in vitro susceptibility than imipenem [22]. Evidence from experimental studies using antimicrobial combinations has demonstrated synergy in vitro, which has also been supported by clinical observation with imipenem-amikacin, imipenem-cefotaxime and amikacin-cefotaxime [23, 24].

The duration of therapy required is unknown, but reports in the literature recommend 6 weeks in localized forms of nocardiosis and 6 months to $1 \mathrm{yr}$ in disseminated nocardiosis [25-27].

In the present series, we observed resolution in six patients, three with localized and three with disseminated disease. Evolution in localized disease was normalization after 6 weeks of treatment. However, in patient No. 1, antibiotic treatment was prolonged due to his immunodepressed status. Dissemination appeared in the two patients with HIV infection and, surprisingly, in a patient without underlying disease. In spite of that, after 6 months of antibiotic treatment, Nocardia had been eradicated without subsequent recurrence. A trend towards chronicity was observed in one case (patient No. 7), with multiple recurrent episodes of infection, confirmed by identical antibiotic susceptibility studies. This peculiar case had a chronic pulmonary disease (bronchiectasis), and Nocardia that was resistant to TMP-SMX. The patient received successive treatments with aminoglucosides combined with imipenem or ciprofloxacin, and showed apparent cure with recurrences. Some authors have observed the persistence of L-forms within macrophages in vitro, but the relationship between this finding and recurrences of the disease has not been proved [28].

Evolution was fatal in three patients. Prognostic factors related to high mortality were associated with corticosteroid treatment and dissemination of the infection [4]. In the present series, mortality in the group of patients receiving corticosteroid treatment was high (2 out of 4 ), compared to the group not receiving such treatment (1 out of 6). Mortality has also been associated with dissemination, especially when the central nervous system is involved (80\%) [3, 4], as was the case in the present series. However, the effect of corticosteroids and other possible factors, such as underlying disease or antinocardial antibiotic therapy, did not appear to influence the local or systemic spread of the disease [3, 4]. A similar number of patients with or without corticosteroid treatment was found in localized and disseminated disease.

In summary, four general conclusions can be made. Firstly, pulmonary nocardiosis is difficult to diagnose on the basis of clinical and radiological findings. A high 
level of clinical suspicion is required in patients with risk factors and in whom no other microorganisms have been identified. Microbiologists must be informed, in such cases, to include specific stains and cultures to investigate the presence of Nocardia spp. Secondly, species classically identified as Nocardia asteroides complex may contain five or more distinct groups, with major differences in their pathogenicity and invasive capabilities. Such variation may contribute to the broad clinical spectrum of pulmonary nocardiosis and also explain differences in response to therapy. Thirdly, susceptibility tests should be interpreted conservatively, taking into account that standardization is not achieved. Finally, difficulties in treatment arise due to the reasons discussed above. New alternative drug therapy and combined treatment with proven synergy, such as imipenem and amikacin, for initial therapy can offer some advantage, mainly in immunodepressed patients.

\section{References}

1. Lerner PI. Nocardia species. In: Mandell GL, Douglas RG, Bennett JE, eds. Principles and Practice of Infectious Diseases. 2nd Edn. New York, John Wiley \& Sons, 1985; pp. 192-206.

2. Beaman BL, Burnside J, Edwards B, Causey W. Nocardial infections in the United States, 1972-1974. J Infect Dis 1976; 134: 286-289.

3. Murray JF, Finegold M, Froman S, Will DW. The changing spectrum of nocardiosis. Am Rev Respir Dis 1961; 83: 315-330.

4. Presant CA, Wiernik PH, Serpick AA. Factors affecting survival in nocardiosis. Am Rev Respir Dis 1973; 108: 1444-1448.

5. Bach MC, Sabath CD, Finland M. Susceptibility of Nocardia asteroides to 45 antimicrobial agents in vitro. Antimicrob Agents Chemother 1973; 3: 1-8.

6. Gordon MA. Aerobic pathogenic Actinomycetaceae. In: Lennete EH, Balows A, Hansley WJ, Truant JD, eds. Manual of Clinical Microbiology. Washington DC, American Society for Microbiology, 1980; pp. 180-194.

7. Wallace RJ, Septimus EJ, Musher DM, Martin RR. Disk diffusion susceptibility testing of Nocardia species. $J$ Infect Dis 1977; 135: 568-576.

8. Kirch JA, Stinson EB, Remington JS. Nocardia infection in heart transplantation patients. Ann Intern Med 1975; 82: 18-26.

9. Goltz HA, Lavery DP, Kapila R. Actinomycetales infection in the acquired immunodeficiency syndrome. Ann Intern Med 1985; 102: 203-205.

10. Rosett W, Hodges GR. Recent experiences with nocardial infections. Am J Med Sci 1978; 276: 279-285.

11. Filice GA. Inhibition of Nocardia asteroides by neutrophils. J Infect Dis 1985; 151: 47-56.

12. Deem RL, Doughty FA, Beaman BL. Immunologically specific direct T-lymphocyte-mediated killing of Nocardia asteroides. J Immunol 1983; 130: 2401-2406.

13. Black CM, Beaman BL, Donnovan RM, Goldstein E. Effect of virulent and less virulent strains of Nocardia asteroides on acid-phosphatase activity in alveolar and peritoneal macrophages maintained in vitro. J Infect Dis 1983; 148: 117-124.

14. Peabody JW, Seabury JH. Actinomycosis and nocardiosis. Am J Med 1960; 28: 99-115.

15. Feigin DS. Nocardiosis of the lung: chest radiographic findings in 21 cases. Radiology 1986; 159: 9-14.

16. Kramer MR, Uttamchandani RB. The radiographic appearance of pulmonary nocardiosis associated with AIDS. Chest 1990; 98: 382-385.

17. Tsukamura M. Nocardiae that recently caused lung infection in Japan: Nocardia asteroides and Nocardia farcinica. Microbiol Immunol 1982; 26: 341-345.

18. Smego RA Jr, Gallis HA. The clinical spectrum of Nocardia brasiliensis infection in the United States. Rev Infect Dis 1984; 6: 164-180.

19. Saubolle MA. In vitro susceptibility testing of clinical isolates of Nocardia. Clin Microbiol Newsl 1993; 15: 169-172.

20. Runyon EH. Nocardia asteroides: studies of its pathogenicity and drug sensitivities. J Lab Clin Med 1951; 37: 713-725.

21. Farina C, Boiron P, Goglio A, Provost F. Human nocardiosis in northern Italy from 1982 to 1992: Northern Italy Collaborative Group on Nocardiosis. Scan J Infect Dis 1995; 27: 23-27.

22. Khardori N, Shawar R, Gupta R, Rosenbaum B, Rolston $\mathrm{K}$. In vitro antimicrobial susceptibilities of Nocardia species. Antimicrob Agents Chemother 1993; 37: 882884.

23. Gombert ME, Aulicino TM. Synergism of imipenem and amikacin in combination with other antibiotics against Nocardia asteroides. Antimicrob Agents Chemother 1983; 24: 810-811.

24. Gombert ME, Aulicino TM, du Bouchet L, Silverman GE, Sheinbaum WM. Therapy of experimental cerebral nocardiosis with imipenem, amikacin, trimethoprimsulfamethoxazole, and minocycline. Antimicrob Agents Chemother 1986; 30: 270-273.

25. Curry WA. Human nocardiosis: a clinical review with selected case reports. Arch Intern Med 1980; 140: 818826.

26. Byrne E, Brophy BP, Perrett LV. Nocardia cerebral abscesses: new concepts in diagnosis, management and prognosis. J Neurol Neurosurg Psychiatry 1979; 42: 10381045.

27. Fernandez-Guerrero ML, Torres A, Díaz Curiel M, Soriano F. Successful treatment of nocardial thigh abscess and possible brain abscess with co-trimoxazole. Eur $J$ Clin Microbiol 1985; 4: 430-431.

28. Jonsson S, Wallace RJ Jr, Hull SI, Musher DM. Recurrent Nocardia pneumonia in an adult with chronic granulomatous disease. Am Rev Respir Dis 1986; 133: 932-934. 